\title{
Tools and publications for reference decay data
}

\author{
C. Dulieu, M.M. Bé, and V. Chisté \\ Laboratoire National Henri Becquerel, LNE-CEA/LNHB, CEA Saclay, 91191 Gif-sur-Yvette Cedex, France
}

\begin{abstract}
As a primary laboratory in the field of ionizing radiation metrology, the LNE-CEA/LNHB is involved in measurement, evaluation and dissemination of radioactive decay data.

Data measurements obtained by various laboratories are evaluated by an international group of experts (Decay Data Evaluation Project) in order to establish a set of recommended decay scheme data. Those data are subsequently distributed to users through various tools developed in our laboratory and well suited to each application domain. They are presented in an exhaustive and documented way through the publication of a multi-volume printed monograph (Monographie BIPM-5), for which a new issue has been released by the end of 2006. This monograph is recommended as a reference database by the CCRI (Comité Consultatif des Rayonnements Ionisants) of the BIPM. Those data can also be accessed via a specific software NucLérDe whose database contains more than 300 nuclides of particular interest.

In the field of alpha and gamma spectrometry, a web application has been specially developed in order to present the major emissions of a given nuclide. Moreover, it allows to find nuclides corresponding to search criteria on their emission energies or intensities.

Finally a booklet version of this database will soon be published. It will present in a concise way the main alpha, X, gamma and electron emissions and their half-life, for some 250 nuclides.

This paper intends to describe some features of these tools and publications.
\end{abstract}

\section{Introduction}

The evaluation of nuclear decay data in the Laboratoire National Henri Becquerel (LNHB) in CEA began in 1974 and many publications on this topic were published since this time. In order to facilitate the management and distribution of these data, a computerized database called NucLÉrDE was developed as well as many tools for both evaluators and users.

Thanks to the data collected in this unique database NuclérDE used as an entry point, and the development of tools such as the SAISINUC program to maintain and update this base, the LNHB is able to offer a variety of tools related to reference nuclear decay data for users in the fields of ionizing radiation metrology, nuclear medicine, monitoring and reactor shielding, spectrometry and environmental studies. We present hereafter some of the tools and publications which are distributed.

\section{Data evaluation tools}

Evaluators of the DDEP (Decay Data Evaluation Project) working group [1] use a more powerful version of the NucLÉIDE application (described hereafter) called SAISINUC to insert data in the Nuclérde database and offers various checking tools such as level balance, energy conservation, internal conversion coefficients calculation, etc. This software is also interfaced with other useful applications like ICC99v3a [2] (calculation of internal conversion coefficients), LWEIGHT [3] (statistical analysis of data sets), Emission [4, 5] (calculation of X-ray and Auger electrons emissions), etc. in order to obtain a consistent set of data for the nuclide being evaluated.

After a review procedure among evaluators, the newly evaluated nuclide can be inserted in the database and then becomes available for the users through various tools based on the NuclérDE database and via printed or electronical publications.

\section{NUCLEIDE CD-Rom}

This is a stand-alone application developed for Windows based PCs using the Microsoft Access database management system. This software contains a database of detailed recommended decay data (and their uncertainties) for about 200 nuclides, and additional alpha and gamma emission data for 200 other nuclides of particular interest in the field of spectrometry. This application offers easy access to the nuclear and atomic decay data through adapted layout windows, as well as a multi-criteria search engine and extraction tools in order to export data to other applications. Decay schemes of comprehensively evaluated nuclides can also be automatically drawn from the data [6].

\section{LNHB web site}

In the atomic and nuclear data section of our website, one can find various information concerning evaluated nuclides, mainly in the form of pdf or text files.

The DDEP works present the latest nuclide evaluations carried out by the members of the Decay Data Evaluation Project working group. This page gives all the recommended data relative to a nuclide and information concerning the way the evaluation was achieved in pdf file, as well as the data in ENSDF file format. As of today, nearly 120 nuclides are proposed to consultation. The web site is regularly updated as soon as a new evaluation becomes available. 


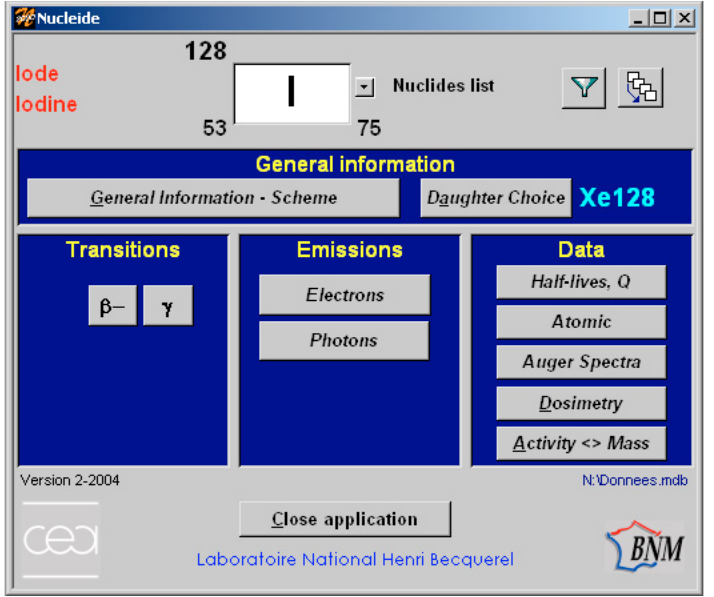

Fig. 1. NucLÉIDE software main window.

Other publications can also be found on the web site, like the Table of Recommended Half-Lives. This table contains nearly 450 nuclides and is updated almost every year.

The LNHB web site can be found at this address:

http://www.nucleide.org/

and the Atomic and Nuclear Data section is available at: http://www.nucleide.org/NucData.htm.

\section{Monographie BIPM-5}

All the latest evaluated nuclides are periodically published in a printed monograph under the auspices of BIPM (Bureau International des Poids et Mesures) [7], as well as electronically in a pdf file format available on CD-Rom or on the BIPM web site. The latest publication (edited in december 2006) is the third volume in the series and contains 28 nuclide evaluations as well as the comments on how these evaluations were conducted [8].

\section{MiniTable}

The LNHB will soon publish (end of 2007) a new and completely revised edition of its booklet version of the Table of Radionuclides known as "MiniTable" [9]. This work will present in a concise way the main alpha, X, gamma and electron emissions, as well as half-life and daughters for a selection of about 250 nuclides of particular interest in the field of spectrometry and practical applications.

\section{Laraweb/NUCLEIDE-LARA}

As indicated by its name, Laraweb is a web-based application designed for spectrometry purposes. It is derived from the previous version LARA, distributed on floppy and paper tables [10]. It allows to find information regarding about 400 nuclides of particular interest in the field of alpha, Xand gamma-ray spectrometry. The main data presented are half-life, daughters, alpha, $\mathrm{X}$ and gamma emissions for each selected nuclide.

Trough an integrated search engine, one can retrieve all nuclides corresponding to a range of chosen search criteria

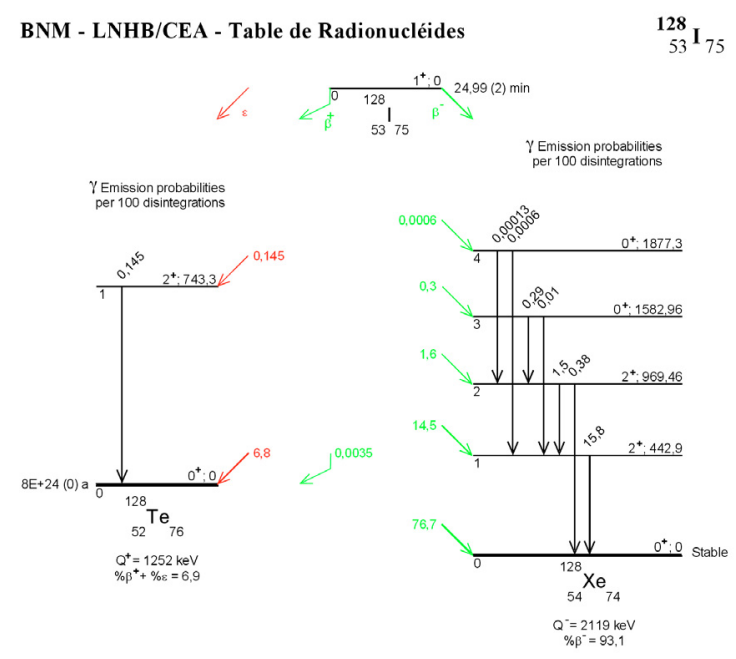

Fig. 2. NuCLÉIDE decay scheme is drawn automatically from the data present in the database.

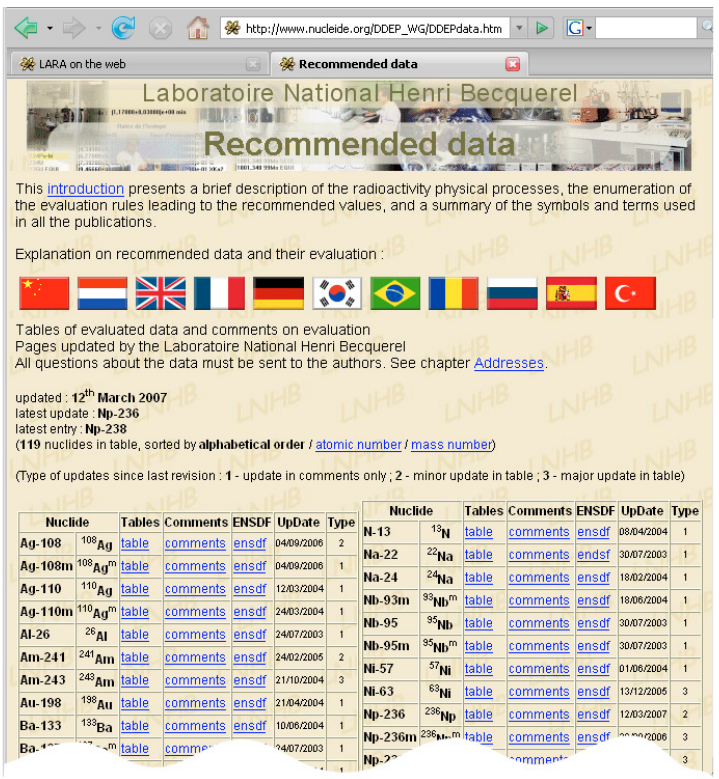

Fig. 3. DDEP recommended decay data available on LNHB web site (extract).

such as emission type (alpha or photons), emission energy, emission intensity, mass number and half-life. Energy and intensity thresholds can also be set in order to show only the main emissions of a nuclide.

This tool can be useful to quickly identify the origin of an alpha or gamma ray in a spectrum, knowing the energy of the peak.

Laraweb can be found on the Atomic and Nuclear Data section of the LNHB web site:

http://www.nucleide.org/NucData.htm or directly at the following address: http://laraweb.free.fr/.

\section{DDEP Group}

The current and past participants in the DDEP evaluation works are V.P. Chechev and N. Kuzmenco (Russia), 


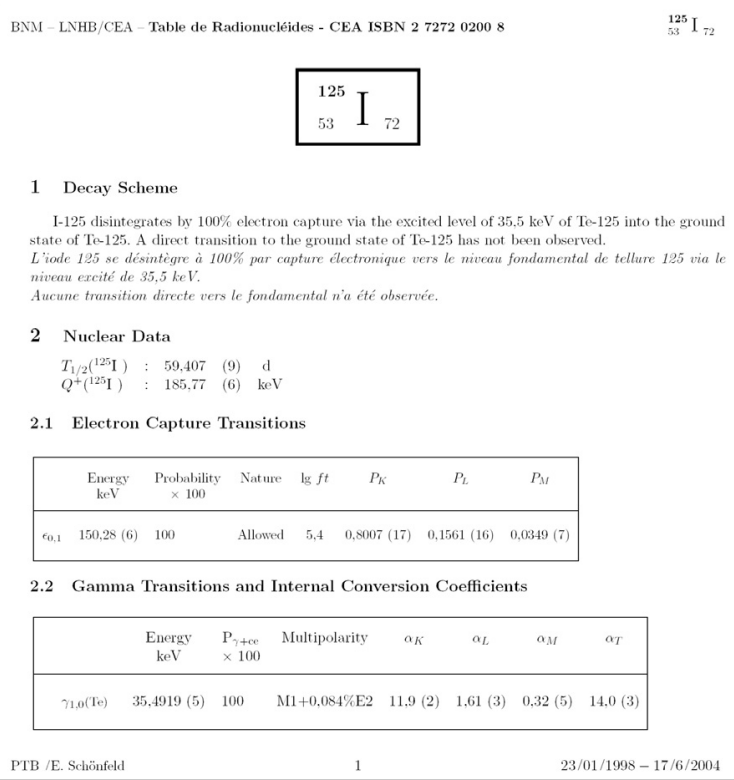

Fig. 4. Example of nuclide data presented in Monographie BIPM-5. Spectrum shown in fig. 2 is also included in this publication.

\begin{tabular}{|c|c|c|c|}
\hline${ }_{53}^{128}$ I & \multicolumn{3}{|c|}{$T_{1 / 2}: 24,99(2) \min$} \\
\hline \multicolumn{4}{|c|}{ Daughter(s): $\left(\beta^{+}, \varepsilon\right)$ Te-128, ( $(\beta)$ Xe-128 } \\
\hline \multicolumn{4}{|c|}{ Electrons $(6$ lines $)-\Sigma\left(l_{\mathrm{e}}\right)$ omitted: $1,1 \%$} \\
\hline Energy (keV) & Intensity (\%) & Type & Origin \\
\hline 3,55 & 5,9 & Auger $\mathrm{L}$ & - Te-128 \\
\hline \multicolumn{4}{|c|}{ Beta - $(5$ lines $)-\Sigma\left(1_{\beta}-\right)$ omitted: $0,30 \%$} \\
\hline $\mathrm{E} \max .(\mathrm{keV})$ & \multicolumn{3}{|c|}{ E avg. (keV) Intensity (\%) } \\
\hline 1150 & 408,8 & \multicolumn{2}{|c|}{1,6} \\
\hline 1676 & 635,7 & \multirow{2}{*}{\multicolumn{2}{|c|}{$\begin{array}{l}14,5 \\
76,7\end{array}$}} \\
\hline 2119 & 834,1 & & \\
\hline \multicolumn{4}{|c|}{$\mathbf{X}$ (10 lines) $-\Sigma(\mathrm{I})$ omitted: $0,18 \%$} \\
\hline Energy (keV) & Intensity (\%) & Type & Origin \\
\hline 4,13 & 0,46 & $x_{L}$ & Te-128 \\
\hline 27,2 & 1,45 & $x_{\mathrm{K} \alpha 2}$ & Te-128 \\
\hline 27,47 & 2,7 & $\mathrm{X}_{\mathrm{K} \alpha 1}$ & Te-128 \\
\hline 31,1 & 0,78 & $x_{K \beta 1}$ & Te-128 \\
\hline 31,76 & 0,16 & $x_{k \beta 2}$ & Te-128 \\
\hline \multicolumn{4}{|c|}{ Gamma (9 lines) - $\Sigma\left(l_{\gamma}\right)$ omitted: $0,8 \%$} \\
\hline Energy (keV) & Intensity $(\%)$ & Type & Origin \\
\hline 442,9 & 15,8 & $\gamma$ & $\mathrm{Xe}-128$ \\
\hline 526,56 & 1,5 & $\gamma$ & $\mathrm{Xe}-128$ \\
\hline \multicolumn{4}{|c|}{ Production mode Possible impurities } \\
\hline \multicolumn{4}{|c|}{$\mathrm{I}-127(\mathrm{n}, \gamma) \mathrm{l}-128 \quad$ none } \\
\hline Reference: $\mathrm{LNE}$ & -CEA/LNHB - 19 & & \\
\hline
\end{tabular}

Fig. 5. Extract of the MiniTable (draft version).

E. Browne, C. Baglin and F. Kondev (USA), A. Nichols (Austria), T.D. MacMahon (United Kingdom), K.B. Lee (South Korea), E. Schönfeld (retired, Germany), M.M. Bé, V. Chisté and C. Dulieu (France). Special mention is dedicated to R.G. Helmer, who died two years ago, and was one of our mainsprings in this group.

\section{Conclusion}

Data evaluation and dissemination is a constant process in which the LNHB is deeply involved in cooperation with the DDEP group. We are permanently updating data and tools and

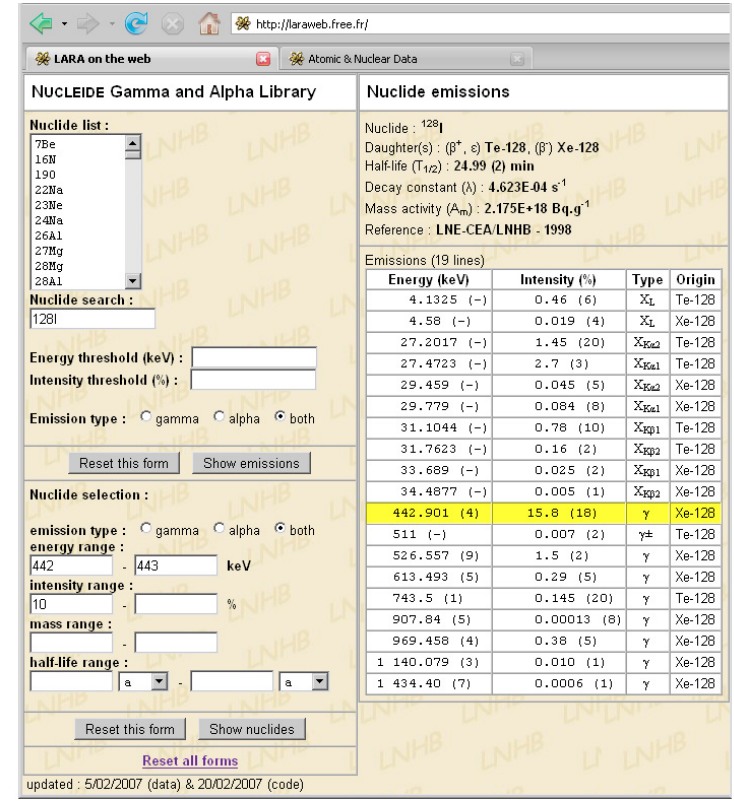

Fig. 6. Example of information that can be found through the Laraweb application.

developing new ones to fulfil the needs of the users and the evaluators.

\section{References}

1. R.G. Helmer, E. Browne, M.M. Bé, J. Nucl. Sci. Technol. Suppl. 2, 455 (2002).

2. V.M. Gorozhankin, N. Coursol, E.A. Yakushev, Ts. Vylov, C. Briançon, Appl. Radiat. Isot. 56, 189 (2002).

3. M.J. Woods, A.S. Munster, National Physical Laboratory Report RS(EXT) 95 (1988).

4. E. Schönfeld, H. Janßen, Nucl. Instrum. Meth. Phys. Res. A 369, (1996) 527.

5. M.M. Bé, V. Chisté, C. Dulieu, Appl. Radiat. Isot. 64, 1435 (2006).

6. M.M. Bé, E. Browne, V. Chechev, V. Chisté, R. Dersch, C. Dulieu, R.G. Helmer, N. Kuzmenco, A.L. Nichols, E. Schönfeld, Nucléide, Table de Radionucléide sur CD-Rom, Version 2, CEA/BNM-LNHB, 91191 Gif-sur-Yvette, France, 2004.

7. M.M. Bé, E. Browne, V. Chechev, V. Chisté, R. Dersch, C. Dulieu, R.G. Helmer, T.D. MacMahon, A.L. Nichols, E. Schönfeld, Table of Radionuclides, Monographie BIPM5, ISBN 92-822-2207-7 (set) and ISBN 92-822-2205-5 (CD), CEA/BNM-LNHB, 91191 Gif-sur-Yvette, France and BIPM, Pavillon de Breteuil, 92312 Sèvres, France, 2004.

8. M.M. Bé, E. Browne, C. Baglin,V. Chechev, N. Kuzmenco, V. Chisté, C. Dulieu, R.G. Helmer, F. Kondev, T.D. MacMahon, K.B. Lee, Table of Radionuclides, Monographie BIPM-5, ISSN 92-822-2204-7 (set), ISBN 92-822-2218-7 (Vol. 3) et ISBN 92-822-2219-5 (CD), CEA/LNE-LNHB, 91191 Gif-sur-Yvette, France and BIPM, Pavillon de Breteuil, 92312 Sèvres, France, 2006.

9. MiniTable (EDP Sciences, Les Ulis, 2007).

10. C. Morillon, M.M. Bé, J. Lamé, C. Jean, Nucléide-Lara : Bibliothèque de données nucléaires pour la spectrométrie gamma et alpha, Vol. 1 et 2, ISBN 2-7272-0212-1 and 2-7272-0213-X, CEA/DAMRI, 91191 Gif-sur-Yvette, France, 2000. 